\title{
Study of the Weights and Dimensions of the Body in the Iraqi Awassi Sheep
}

\author{
Yousif H. Al.Saltani \\ Muthanna S. Azzawi \\ Al-Furat Al-Awst Technical University, Al-Musaib Technical College , , 51009 \\ Babylon, Iraq \\ m.sabah41@yahoo.com
}

\begin{tabular}{l}
\hline ARTICLE INFO \\
\hline Submission date: $13 / 8 / 2018$ \\
Acceptance date: $5 / 9 / 2018$ \\
Publication date: $10 / 3 / 2019$ \\
\hline
\end{tabular}

\section{Abstract}

This study was undertaken at the native feilds of the sheep farms in Sadet Al-hindia, Babylon Governorate, over period from December 2017 to March 2018. The aim of this was study the effect of type of birth and sex of lambs on the traits of birth and weaning weight, body dimensions(body length, chest circumference, height at the front and back)in awassi sheep. the results showed that significant superiority $(\mathrm{p}<0.05)$ for twins lambs $(40.9 \mathrm{~cm})$ on single lambs $(39.33 \mathrm{~cm})$ in body length at the birth .for the sex of lambs, male lambs has significant superiority $(p<0.05)$ on female lambs in heightin front $(41.03,40.07 \mathrm{~cm}$ Respectevly), but the type of birth and sex of lambs had nonsignificant effect of other parameters .

Key words: Awassi sheep, Sex of the baby, Birth weight, Type of birth.

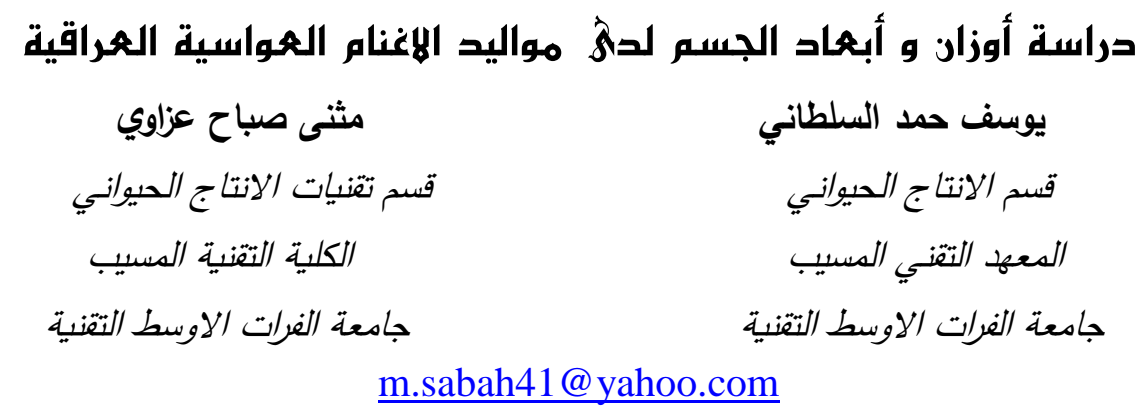

اجريت الدراسة في حقل القطاع الخاص لتربية الاغنام في منطقة سدة الهندية - محافظة بابل للمدة من كانون

الاول2017 ولغاية اذار 2018, لدراسة تأثير نوع الولادة وجنس المولود في صفات الوزن عند الميلاد والوزن عند الفطام وابعاد الجسم

التي شملت ( طول الجسم و محيط الصدر و الارتفاع عند المقدمة و الارتفاع عند المؤخرة ) لدى الاغنام العواسية العراقية. أظهرت

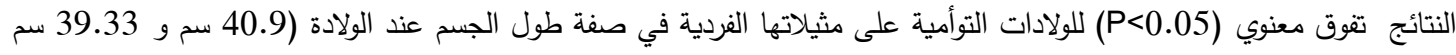

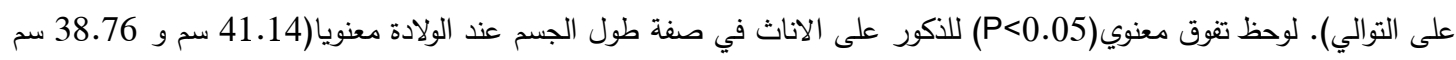

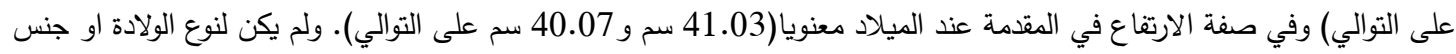
الحملان اي تأثير معنوي في بقية الصفات المدروسة. الكلمات الدالة: الاغنام العواسية , جنس المولود , الوزن عند الميلاد , نوع الولادة . 


\section{المقدمة}

ويعد الاهتمام بالتراكيب الوراثية الحديثة أمراً ضرورياً لتوفير الاحتياجات المستقبلية من اللحوم والحليب والصوف ولذلك يجب اخذ الاحتياطات للتتبؤ بالإنتاج من جوانب وراثية قادرة على الاستجابة السريعة لبيئة

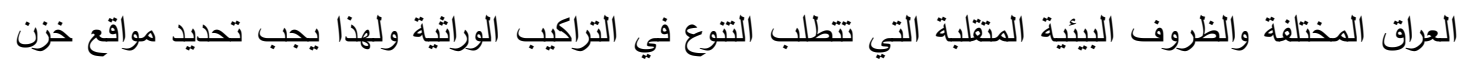
وراثية (بنك جينات) لمعرفة التراكيب الوراثية ذات القدرة على مقاومة الظروف المختلفة ومعرفة انتاج الحيوانات على اساس وراثي[1].

يؤدي استعمال عدد محدد من الذكور المتفوقة وراثيا الى ارتفاع معامل التربية الداخلية وهذا يحد من مستويات التتوع الجيني للحفاظ على التتوع عبر الاجيال [2]. من الظروف المهمة للاختيار المناسب للأغنام

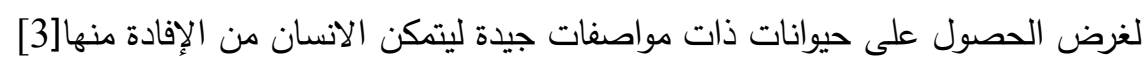

اهتمَ الباحثون ببعض القياسات للحملان عند الولادة وعند الفطام وكذلك في اعمار مختلفة وذلك للحصول

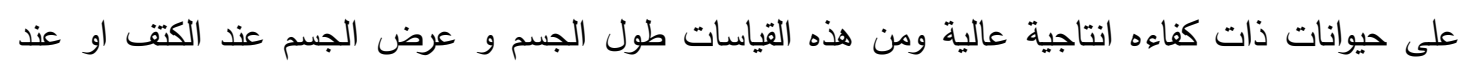
الحوض و ارتفاع الجسم عند المقدمة او المؤخرة و محيط الفخذ و محيط البطن و محيط الصدر و والمسافة بين الخط الظهري والبطني للحيوان وغيرها وتوجد هناك عوامل عديدة تؤثر في وجود فروقات كبيره في قطيع الاغنام لهذه القياسات من التراكيب الوراثية والسلالة والجنس ونوع الولادة موسم الولادة والتغذية وطريقة التربية والادارة والامراض والظروف البيئية التي يعيش بها القطيع، لذلك يجب حساب تأثير الظروف غير الوراثية والحد من لتن

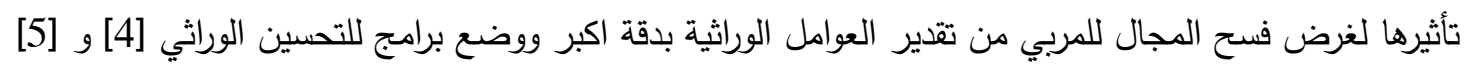
لذا فأن الهدف من هذه الدراسة تحديد تأثير نوع الولادة وجنس المولود في صفات الوزن عند الميلاد والفطام وابعاد الجسم في الاغنام العواسية العراقية.

المواد وطرائق العمل

$$
1
$$

اجريت الدراسة في محطة القطاع الخاص في سدة الهندية على 45 نعجة ومواليدها (60 مولود) من الاغنام العواسية للمدة من كانون الاول 2017 ولغاية اذار 2018 لموسم انتاجي واحد و تراوحت العمار النعاج من 2 الى 8 سنوات وجُمعت بيانات الاغنام المستخدمة في التجربة بمعرفة اعمار الحيوانات وانواعها من وند سجلات المحطة و انجز الجزاء الوراثي في مختبر الوراثة الجزيئية.

2

ربيت الاغنام في حظائر شبة مفتوحة (40\% مسقفه و60\% مفتوحه) مخصصه لإيواء الاغنام وتبلغ مساحة الحظيرة (50 م 15 × م) تتوافر فيها مناهل بطول 15 م ومعالف بطول 30 م للتغذية المركزة. توضع فئس

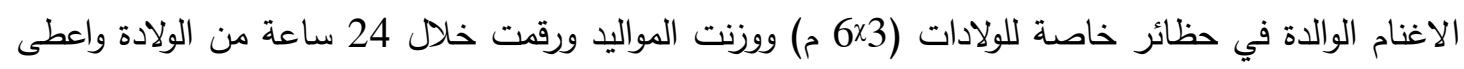

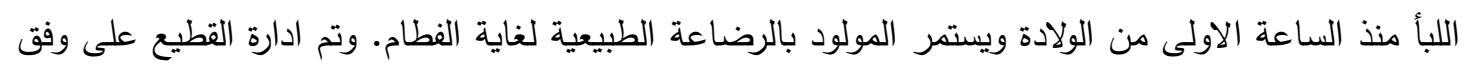


برنامج يتضمن التغذية والتحضير لموسم السفاد والاعداد لمرحلتي الحمل و الولادة فضلاً عن الرعاية الصحية والبيطرية.

$$
3
$$

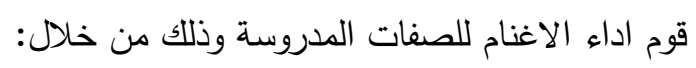
- اخذت اوزان الجسم للمواليد عند الولادة والفطام عند هذين العمرين باستخدام ميزان خاص لذاص للك.

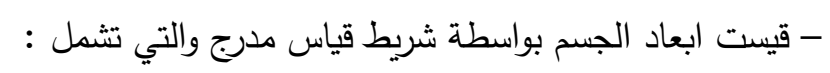

أ - ارتفاع الجسم عند المقدمة وقيست المسافة من نقطة اتصال الرقبة بالجسم الى الارض لهئ (وهي تمثل

$$
\text { ارتفاع الجسم عند المقدمة). }
$$

ب - ارتفاع الجسم عند المؤخرة : قيست المسافة من نقطة نهاية الظهر الى الارض (وهي تمثل ارتفاع

$$
\text { الجسم عند المؤخرة). }
$$

ج - محيط الصدر : قيست هذه المنطقة بلف الشريط المدرج حول منطقة الصدر خلف الارجل الامامية

$$
\text { مباشرةً. }
$$

$$
4
$$

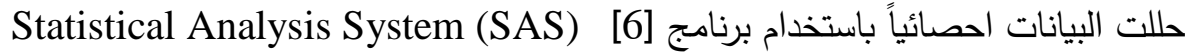
لدراسة تأثير نوع الولادة وجنس المولود في عدد من صفات النمو وقورنت الفروقات المعنوية بين المتوسطات $\mathbf{Y i j k}=\mathbf{M}+\mathbf{G i}+\mathbf{P j}+\mathbf{e i j k}$ بأستخدام طريقة المربعات الصغرى (Least Sqare Means) K قيمة المشاهدة Yijk M Gi جنس : Pj

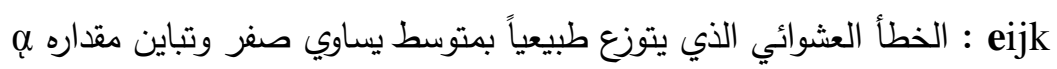

\section{النتائج والمناقشة}

نوع الولادة : يتضح من جدول (1) بعدم وجود فروقات معنوية لنوع الولادة (مفرده او توأمية) بين صفات الوزن وابعاد الجسم عند الميلاد، في حين كان معنوياً لصفة طول الجسم (P<0.05) عند الميلاد اذ تفوقت الولادات التوأمية على الولادات الفردية والتي بلغت 40.93 × 0.80 و 39.30 × 0.71 سم على التوالي. وقد يعود السبب في عدم وجود فروقات معنوية الى وجود الدفع الغذائي الجيد للأمهات اثناء مدة الحمل وقبلها مما يؤدي

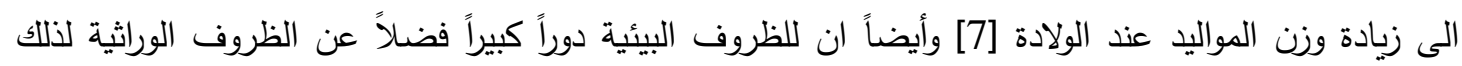
يجب الاهتمام بالظروف البيئية لما لها من أثر مهم في دقة وتقدير المعالم الوراثية في برامج الانتخاب للصفات 
الانتاجية [8] اما الاوزان الجيدة للأمهات فيعطي وزن افضل عند الولادة وان لوزن الام تأثيراً معنوياً في وزن

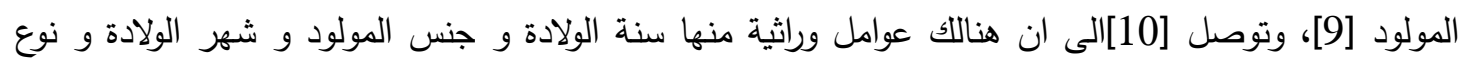
الولادة كان لها تأثير معنوي (P<0.05) في صفات النمو المدروسة للتراكيب الوراثية المختلفة واشار كذلك الى ولى

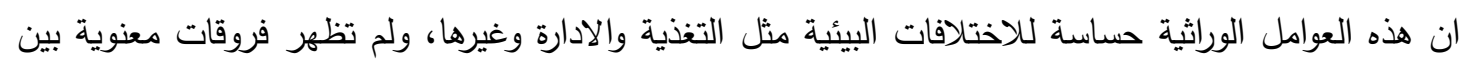

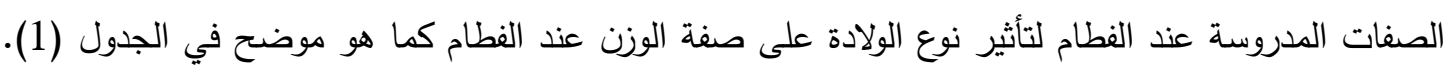

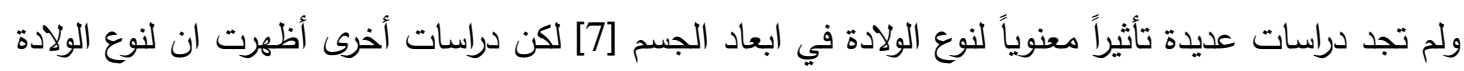
تأثيراً معنويا لصالح الولادات الفردية على الولادات التوأمية في الوزن عند الميلاد والفطام[11:

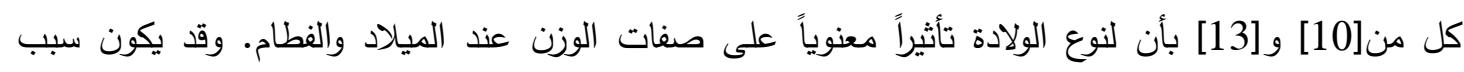

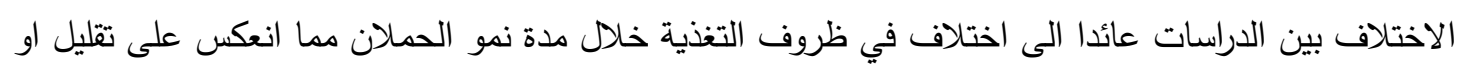

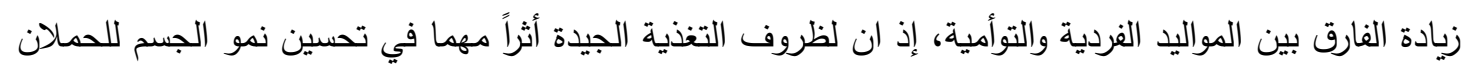

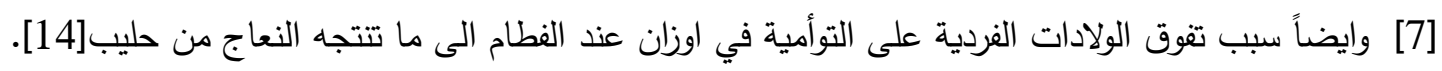

جدول 1 : متوسطات المربعات الصغرى \pm الذطأ القياسي للصفات قيد الدراسة عند الميلاد لتأثير نوع الولادة لاى الاغنام العواسية العراقية.

\begin{tabular}{|c|c|c|c|}
\hline \multirow[t]{2}{*}{ مستوى المعنوية } & \multicolumn{2}{|c|}{ المتوسط العام \ الخطأ القياسي } & \multirow[t]{2}{*}{ 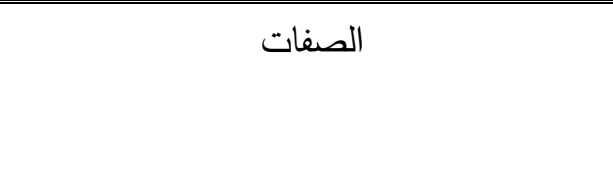 } \\
\hline & توأمية (العدد = 30) & فردية (العدد = 30) & \\
\hline NS & $0.07 \pm 4.95$ & $0.05 \pm 4.95$ & الوزن عند الميلاد (كغم) \\
\hline NS & $0.44 \pm 17.36$ & $0.41 \pm 18.50$ & الوزن عند الفطام (كغم) \\
\hline NS & $0.43 \pm 12.41$ & $0.40 \pm 13.54$ & معدل الزيادة الوزنية بين الميلاد والفطام (كغم) \\
\hline * & a $0.80 \pm 40.93$ & b $0.71 \pm 39.30$ & طول الجسم عند الميلاد (سم) \\
\hline NS & $0.38 \pm 50.40$ & $0.68 \pm 51.23$ & طول الجسم عند الفطام (سم) \\
\hline NS & $0.61 \pm 44.26$ & $0.41 \pm 43.13$ & محيط الصدر عند الميلاد (سم) \\
\hline NS & $0.67 \pm 57.87$ & $0.52 \pm 58.97$ & محيط الصدر عند الفطام (سم) \\
\hline NS & $0.34 \pm 40.70$ & $0.37 \pm 40.53$ & الارتفاع من المقدمة عند الميلاد (سم) \\
\hline NS & $0.38 \pm 40.93$ & $0.44 \pm 40.36$ & الارتفاع من المؤخرة عند الميلاد (سم) \\
\hline NS & $0.34 \pm 50.47$ & $0.55 \pm 50.43$ & الارتفاع من المؤخرة عند الفطام (سم) \\
\hline
\end{tabular}




$$
\text { . NS ، (P<0.05) * }
$$

المتوسطات التي تحمل حروف مختلفة ضمن الصف الواحد تختلف معنويا فيما بينها.

\section{جنس المولود}

لم تكن هناك فروق معنوية بين صفات الوزن وابعاد الجسم لتأثير جنس المولود عند الولادة ماعدا صفة

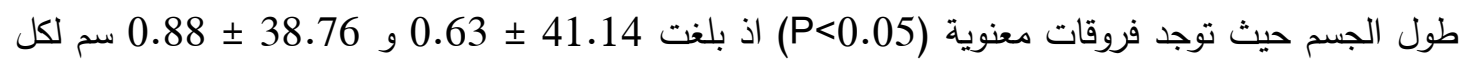
من الذكور والاناث على التوالي وكذللك صفة الارتفاع عند المقدمة توجد فيها فروق معنوية (P>0.05) حيث (P) حيث

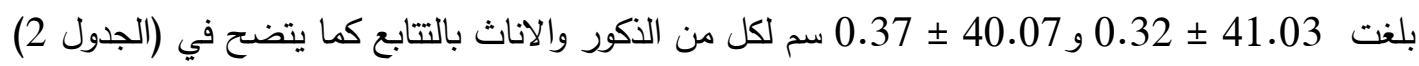

جدول2 : متوسطات المربعات الصغرى 土 الخطأ القياسي للصفات المدروسة لتأثير جنس المولود لاى الاغنام العواسية العراقية.

\begin{tabular}{|c|c|c|c|}
\hline \multirow[t]{2}{*}{ مستوى المعنوية } & \multicolumn{2}{|c|}{ المتوسط العام 土 الخطأ القياسي } & \multirow[t]{2}{*}{ 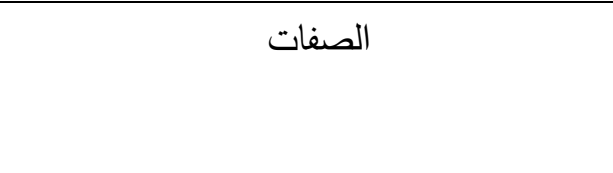 } \\
\hline & اناث (العدد = 26) & ذكور (العدد = 34) & \\
\hline NS & $0.07 \pm 4.90$ & $0.06 \pm 4.99$ & الوزن عند الميلاد (كغم) \\
\hline NS & $0.48 \pm 17.98$ & $0.40 \pm 17.89$ & الوزن عند الفطام (كغم) \\
\hline NS & $48.0 \pm 13.07$ & $0.39 \pm 12.90$ & معدل الزيادة الوزنية بين الميلاد والفطام (كغم) \\
\hline$*$ & b $0.88 \pm 38.76$ & a $0.63 \pm 41.14$ & طول الجسم عند الميلاد (سم) \\
\hline NS & $0.61 \pm 50.34$ & $0.51 \pm 51.17$ & طول الجسم عند الفطام (سم) \\
\hline NS & $0.63 \pm 43.19$ & $0.43 \pm 44.08$ & محيط الصدر عند الميلاد (سم) \\
\hline NS & $0.68 \pm 58.65$ & $0.55 \pm 58.23$ & محيط الصدر عند الفطام (سم) \\
\hline * & b $0.37 \pm 40.07$ & a $0.32 \pm 41.03$ & الارتفاع من المقدمة عند الميلاد (سم) \\
\hline NS & $0.44 \pm 50.73$ & $0.46 \pm 50.23$ & الارتفاع من المؤخرة عند الفطام (سم) \\
\hline & & (PS $)$ (P< & \\
\hline
\end{tabular}


المتوسطات التي تحمل حروف مختلفة ضمن الصف الواحد تختلف معنويا فيما بينها.

وايضاً لم يكن لجنس المولود تأثيرُ معنويٌّ في صفات الوزن وابعاد الجسم عند الفطام وقد يعود السبب في عدم وجود فروقات معنوية بين الجنسين الى ظروف التغذية الجيدة للأمهات قبل واثثاء مدة الحمل وللمواليد بعد الولادة مما ادى الى تلاشي الفروقات بين الجنسين وقد اشار عدد من الباحثين الى ان التغذية الجيدة لمدة طويلة

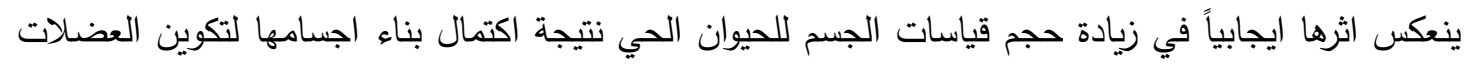

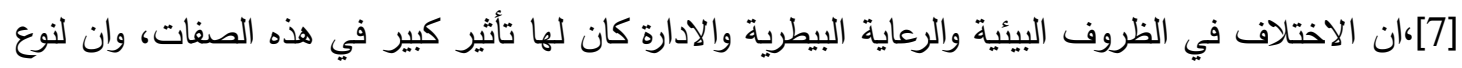

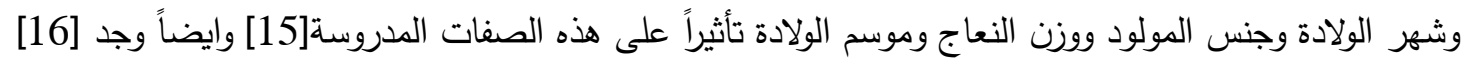
هناك تأثير للعمر والجنس على بعض ابعاد الجسم للحملان العواسي، وبين [17] الى عدم وجود تباين معنوي التي لجنس الحملان والمجموعة الوراثية على صفة الوزن عند الولادة. ولم يلاحظ عدد من الباحثين تأثيراً معنوياً

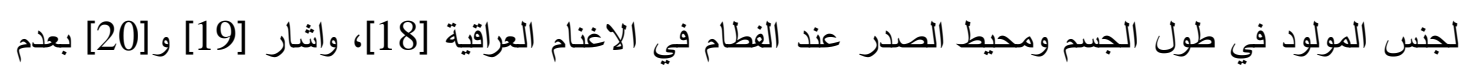

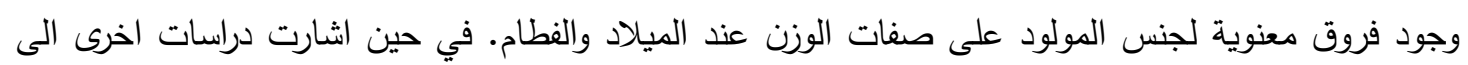
تفوق الذكور على الاناث في ابعاد الجسم [21]، كما بين بعض الباحثين ان لجنس المولود فروقات معنوية

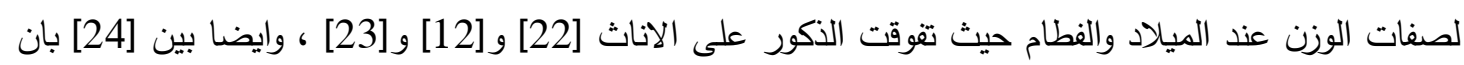
لصفة الوزن عند الولادة يتأثر بجنس المولود ونوع الولادة وعمر النعاج.

الاستنتاجات

ان النتائج التي حصلنا عليها في هذه الدراسة والتي اظهرت عدم وجود فروقات معنوية بين الولادات الفردية

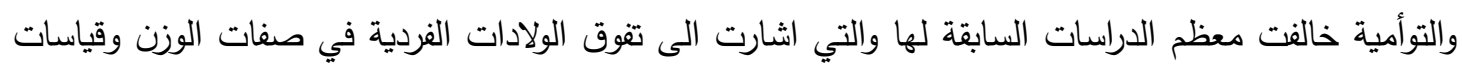

ان هذه النتيجة تشير بوضوح الى اهمية الاعتناء بالأمهات قبل الولادة وبعدها وتوفير الاعلاف اللازمة لذلك

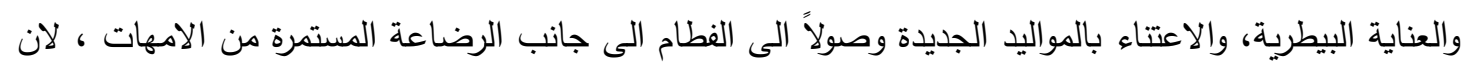

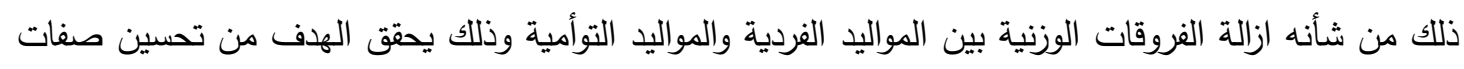
الخصب لدى الاغنام التي هي ابرز ما يبحث عن المربين لتحسين الاداء الانتاجي للأغنام وبالتالي زيادة المدخلات المالية.

CONFLICT OF INTERESTS.

There are non-conflicts of interest .

المصادر

1. Plug, I., and Badenhorst, S. 2001. The Distribution of Macro mammals in South Africa over the past 30000 years. Transvaal Museum, Pretoria Portugal assessed by microsatellites. Journal of animal science 86:2496.

2. Li, M., Tapio, I., Vilkki, J., Ivanova, Z., Kiselyova, T., Marzanov, N., et al. 2007. The genetic structure of cattle populations (Bos taurus) in northern 
Eurasia and the neighbouring Near Eastern regions: implications for breeding strategies and conservation. Molecular Ecology 16: 3839 - 3853.

3. Stolze, M. and Lampkin, N. 2009. Policy for organic farming: rationale andconcepts. Food Policy 34: 237 - 244.

4. Jawasreh, K.I. 2003. Genetic evaluation of Damascus goats in Jordan. Ph.D. Thesis, University of Baghdad, Iraq.

5. Hermiz, H.N., Al-Kass, J.E., Hobi, A.A. and Asofi, M.K. 2009. Genetic and phenotypic parameters of body weights in Iraqi Local Goat and their Crosses with Damascus. J. Duhok Univ., 2(1): 189 - 194.

6. SAS. 2012. Statistical Analysis System, users guide. Statistical. Version 9.1th ed .SAS. Inst .Inc. Cary. N.C. USA.

7.شمس الدين، قصي زكي. 1992. تأثير نظام طول فترة التغذية على النمو وبعض صفات ذبائح الحملان

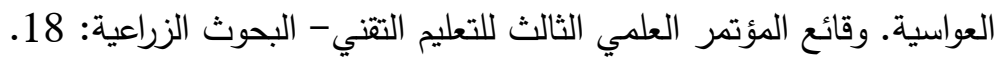

8. Sireli, H.D., Vural, M.E., Karatas, A., Akca, N., Koncaul, S. and Tekel, N. 2015. Birth and weaning weights of Awassi lambs raised in the GAP Intemational Agricultural Research and Training Center. Ankara Univ Vet Fak Derg, 62: 139 - 145.

9.البرزنجي، يوسف محمد صالح نوري. 2003. دراسة النمو وابعاد الجسم للحملان والتقويم الوراثي لإنتاج

الحليب في النعاج الحمدانية. رسالة ماجستير . كلية الزراعة-جامعة صلاح الدين.

10.خلف، احمد اسماعيل وسعيد، سعد ابراهيم وادريس، سجاد مزيد.2010.دور بعض العوامل البيئية

والوراثية في صفات النمو للى حملان العواسي التركي والمحلي والمضرب. مجلة العلوم الزراعية

$$
\text { العراقية، 41(3): } 12 \text { - } 22 .
$$

11. Owen, J. B.1976. Sheep production. Bailliere Tindal, London.

12. Momoh, O.M., Rotimi, E.A. and Dim, N.I. 2013. Breed effect and non-genetic factors effecting growth performance of sheep in a semi -arid region of Nigeria. Journal of Applied Biosciences 67: 5302 - 5307.

$$
\begin{aligned}
& \text { 13.رؤوف، سالم عمر ومحمود، كانياو اسماعيل وعبدالكريم، عبدالخالق عبدالقادر ومحم، } \\
& \text { كوكزكيلاني.2017. تقدير افضل تتبؤ خطي غير منحاز (BLUP) للكباش اعتمادا على وزن } \\
& \text { نسلها عند الولادة والفطام. مجلة العلوم الزراعية العراقية. } 48 \text { (6): } 1405 \text { - } 1411 .
\end{aligned}
$$

14. Juma, K. H., Alkass, J. E. and Aldoori, T. S. 1985. Studies on Some Economic Characteristics in Awassi and Arabi Sheep. 1. Birth and Weaning Weight. Wld. Rev. Anim. Prod. 21 (2) : 55- 59.

15. Al-Tarayrah, J.A. and Tabbaa, M.J. 1999. Some factors affecting body weight and dimensions and its adjustment factors for Awassi Lambs in Jordan. Dirasat, Agricultural Sciences 26(2): 168 - 178.

16. Iqbal, Z.M., Javed, K., Abdullah, M., Ahmad, N., Ali, A., Khalique, A., Aslam, N. and Younas, U. 2014. Estimation of body weight from different morphometric measurements in kajli lambs. The Journal of Animal \& Plant Sci, 24(3): 700 - 703.

$$
\begin{aligned}
& \text { 17.سالم، عبدالله حميد. 2016. بعض العوامل الوراثية واللاوراثية المؤثرة في عدد من ابعاد الجسم لدى } \\
& \text { الماعز المحلي والثامي. مجلة كربلاء للعلوم الزراعية. 3(2): } 12 \text { - } 21 . \\
& \text { 18.التميمي، كاظم توفيق ضحي. 1983. تأثير انظمة مختلفة من الفطام ومستويات التغذية اللاحقة على اداء } \\
& \text { الحملان العرابي وامهاتها من الحليب. رسالة ماجستير ـ كلية الزراعة جامعة البصرة. }
\end{aligned}
$$


19.الخزرجي، وسن جاسم والسامرائي، وفاء اسماعيل وعجيل، حمود مظهور.2014. تأثير بعض العوامل

الوراثية واللاوراثية في صفات الخصوبة والنمو للأغنام العواسية. مجلة العلوم الزراعية العراقية،

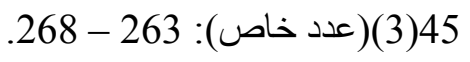

20.احد، بثار ادهم ومهدي، معن فالح وابراهيم، مصطفى خليل ومحمود، الاء شاكر.2615.2015.تأثير سلالة

الام وبعض العوامل اللاوراثية على نمو الحملان قبل الفطام. مجلة ديالى للعلوم الزراعية،7(2):

$$
.19-13
$$

21. Aziz, D. A., Al kass, J. E. and Hermiz, H. N. 1994. Some invironmental and genetic influence on gain in Awassi lambs. IPA J. Agric. Res. 4 (2): 174 184

22. Raoof, S.O., Al-Sherwany, D.O. and Ali, S.S. 2013. Effect of breed, fodder beet and non-genetic factor on the ewe performance in Erbil land Zanco, J. of Pure and Applied Sci. 25(3): 8 - 14.

23. Al-Samarai, W.I., Al-Kazraji, W.J., Al-Jelawi, J.R. and Ajeel, H.M. 2015. The effect of some environmental factor in some growth traits and milk production in Turkish Awassi sheep. Diyila J. Agriculture science 7(2): 5 68.

24. Karakus, F. and Atmaca, M. 2016. The effect of ewe body condition at lambing on growth of lambs and colostral specific gravity, Arch. Anim. Breed, 59: $107-112$. 